\title{
Measuring Spin Relaxation Rates using Satellite Exchange NMR Spectroscopy
}

\author{
Gerard P. Robertson, ${ }^{[\mathrm{a}]}$ Barbara Odell, ${ }^{[\mathrm{a}]}$ Ilya Kuprov,${ }^{[\mathrm{b}]}$ Darren J. Dixon, ${ }^{[\mathrm{a}]}$ and Tim D. W. Claridge ${ }^{* \mathrm{a}]}$
}

\begin{abstract}
An approach to the indirect measurement of nuclear spin relaxation rates of low-magnetogyric ratio (Y) nuclei using the process of satellite exchange is described. The method does not require the observation of, or even the ability to provide radiofrequency pulses to, the low-y nucleus, but requires this to be scalar coupled to an NMR observable nucleus, such as ${ }^{31} \mathrm{P}$ or ${ }^{1} \mathrm{H}$, making it especially attractive for the study of diamagnetic transition metals. In situations where spin relaxation is dominated by chemical shift anisotropy (CSA), the determination of the longitudinal spin relaxation time constant $\left(T_{1}\right)$ of the metal becomes possible, as illustrated for ${ }^{195} \mathrm{Pt}$ and ${ }^{107 / 109} \mathrm{Ag}$.
\end{abstract}

NMR spectroscopy is well-established as the leading technique for the structure elucidation of small molecules and for studying their dynamic behaviour in solution. ${ }^{[1]}$ In particular, spin relaxation rates can be correlated with global and local dynamics within molecules and have been used to report on bond configuration, distance, and rotational correlation times in small molecules. ${ }^{[2]}$ Measurement of the longitudinal relaxation time constants $\left(T_{1}\right)$ for transition metals has the potential to provide valuable information on organometallic complexes relating to their co-ordination geometry, motional properties and molecular interactions. ${ }^{[3]}$ However, little experimental data are available in the literature on the longitudinal relaxation rates of low magnetogyric ratio $(\mathrm{Y})$ transition metal nuclei. ${ }^{[3 b]}$ This is due to a number of factors, including the poor detection sensitivity of low$Y$ nuclei $^{[4]}$ and often the need for specialised NMR probes operating at the low resonance frequencies appropriate to observe (spin-1/2) metals of potential interest such as ${ }^{57} \mathrm{Fe}^{[3 b]},{ }^{89} \mathrm{Y}$, ${ }^{103} \mathrm{Rh},{ }^{[5]}{ }^{107 / 109} \mathrm{Ag},{ }^{[6]}{ }^{183} \mathrm{~W},{ }^{[7]}$ and ${ }^{187} \mathrm{Os}$. These factors make the direct measurement of the relaxation properties of such nuclei largely impossible using standard instrumentation. For example, measurement of relaxation rates for silver $\left({ }^{107 / 109} \mathrm{Ag}\right)$ in the liquid state has been restricted largely to studies of concentrated salt solutions which can exhibit exceedingly long relaxation times (e.g. $971 \mathrm{~s} \mathrm{~T}_{1}$ for $1 \mathrm{M} \mathrm{AgNO}_{3}$ in $\mathrm{H}_{2} \mathrm{O}$ at $90 \mathrm{MHz}\left({ }^{1} \mathrm{H}\right)^{[8]}$ ); such studies are clearly not representative of more highly structured chemical systems.

Herein we demonstrate the use of conventional Exchange

[a] G. P. Robertson, Dr. B. Odell, Prof. D. J. Dixon, Prof. T. D. W. Claridge

Department of Chemistry

University of Oxford

Chemistry Research Laboratory

Mansfield Road

Oxford, OX1 3TA, UK

E-mail: tim.claridge@chem.ox.ac.uk

[b] Prof. I. Kuprov

School of Chemistry,

University of Southampton,

Highfield Campus, Southampton SO17 1BJ, UK

Supporting information for this article is given via a link at the end of the document.
Spectroscopy (EXSY) experiments as a means to study indirectly the relaxation properties of low-y nuclei provided these are scalar coupled to a directly observable nuclide, for example ${ }^{31} \mathrm{P}$ or ${ }^{1} \mathrm{H}$. The method relies only on the ability to observe the high-y spin, without the need to observe, or even pulse, the low$\mathrm{Y}$ nucleus, providing a novel approach to the determination of longitudinal spin relaxation times for nuclei such as transition metals, without the need for specialised NMR instrumentation.

To demonstrate this method for the indirect measurement of relaxation rates, we used a platinum containing complex as a test system since it is also possible to measure directly the relaxation properties of spin- $1 / 2{ }^{195} \mathrm{Pt}$. We chose the non-labile complex cis-dichlorobis(triphenylphosphine) platinum(II) $\mathbf{1}^{[9]}$ in which the metal centre is scalar coupled to readily observable ${ }^{31} \mathrm{P}$ with ${ }^{1} \mathrm{JPt} \mathrm{P}=3682 \mathrm{~Hz}$.

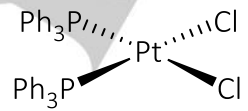

1

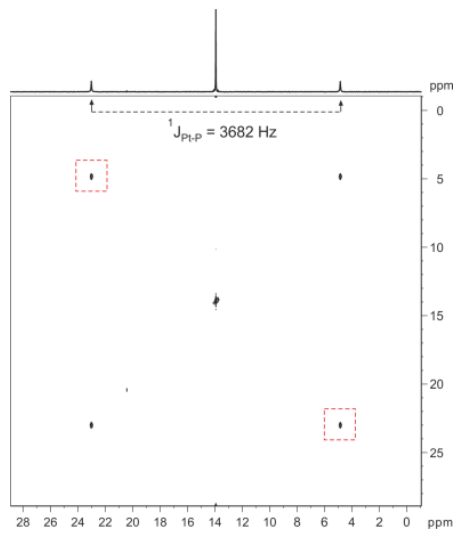

Figure 1. $202 \mathrm{MHz}$ 2-D ${ }^{31} \mathrm{P}_{-}{ }^{31} \mathrm{P}$ EXSY NMR spectrum of $50 \mathrm{mM}$ cisdichlorobis(triphenylphosphine) platinum(II) 1 (mixing time $t_{\text {mix }}=800 \mathrm{~ms}$ ) in $\mathrm{CD}_{2} \mathrm{Cl}_{2}$ at $298 \mathrm{~K}$ showing the ${ }^{195} \mathrm{Pt}$ satellite cross-peaks arising from satellite exchange (boxed in red)

In the proton-decoupled ${ }^{31} \mathrm{P}_{-}{ }^{31} \mathrm{P}$ EXSY spectrum of complex 1, cross-peaks between the ${ }^{195} \mathrm{Pt}$ satellites (33.8 \% total abundance) are clearly visible (Figure 1). The P-Pt bond in $\mathbf{1}$ is not labile and, in accordance with this, no free triphenylphosphine was observed in its ${ }^{31} \mathrm{P}$ spectra, nor was there evidence for the trans isomer being formed. As such, direct chemical exchange of the triphenylphosphine groups cannot be responsible for the appearance of these cross-peaks ${ }^{\dagger}$. Instead, their origin can be ascribed to the inherent longitudinal relaxation of the ${ }^{195} \mathrm{Pt}$ spins occurring during the EXSY mixing period $\left(t_{\text {mix }}=800 \mathrm{~ms}\right.$ for Figure 1$)$. The interchange between nuclear $\alpha-\beta$ spin states of the metal arising from this relaxation during $t_{\text {mix }}$ leads to a corresponding change of the satellite frequency for any ${ }^{31} \mathrm{P}_{-}{ }^{195} \mathrm{Pt}$ spin pair, and hence to the development of cross-peak intensity between the satellites; henceforth we refer to this process as satellite exchange (SE). In

$\dagger$ In principle, these could arise if the triphenyl phosphine ligands were able to dissociate from the metal during the EXSY mixing time and re-complex with another platinum centre having the opposite spin-state. 
accord with this behaviour, the intensities of these cross-peaks display a time-dependent build-up that is dictated by the longitudinal relaxation rates of the platinum spins, even though these are not being directly observed or pulsed. Figure 2 presents the ratio of the integrated peak volumes of the satellite cross-peak to the diagonal peak ( $\left.I_{\text {cross }} / I_{\text {diagonal }}\right)$ against mixing time, $t_{\text {mix }}$. Equivalent results are produced using 1D EXSY measurements and monitoring the peak integral ratio of exchange vs. selectively inverted satellite peak, the 1D approach offering greater time efficiency when individual satellites can be selectively inverted.

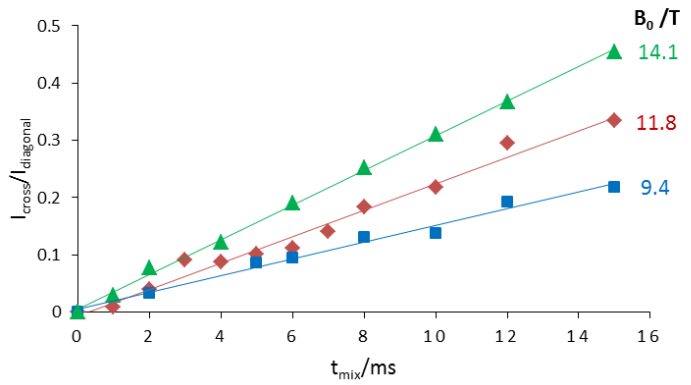

Figure 2. : Time dependence of ${ }^{195} \mathrm{Pt}$-satellite exchange in complex $\mathbf{1}$ at ${ }^{1} \mathrm{H}$ frequencies of $600 \mathrm{MHz}$ (green triangles), $500 \mathrm{MHz}$ (red diamonds) and 400 $\mathrm{MHz}$ (blue squares). Intensities were measured as volume integral ratios of satellite cross-peak: diagonal peak $\left(I_{\text {cross }} / I_{\text {diagonal }}\right)$ from ${ }^{31} \mathrm{P}_{-}{ }^{31} \mathrm{P}$ EXSY spectra recorded at $298 \mathrm{~K}$ in $\mathrm{CD}_{2} \mathrm{Cl}_{2}$.

The relaxation of ${ }^{195} \mathrm{Pt}$ is dominated by the chemical shift anisotropy (CSA) mechanism, ${ }^{[3 a, 10]}$ and hence its relaxation rates exhibit a linear dependence on the square of the static magnetic field strength $\left(B_{0}\right)$. This behaviour is demonstrated for complex 1 in Fig. 2 where the initial-rate profiles for satellite exchange are shown for data recorded at three different field strengths. The linear dependence of the experimentally determined ${ }^{195} \mathrm{Pt}$ relaxation rates (derived as described below) on $\mathrm{B}_{0}{ }^{2}$ is illustrated in Fig $3 \mathrm{a}$, which confirms the dominance of the CSA mechanism on platinum relaxation and strongly evinces the satellite exchange process as reporting indirectly on the metal spin relaxation rates. The CSA-induced broadening of platinum satellites as a function of field strength is well known, ${ }^{[11]}$ and although the appearance of cross-peaks between metal satellites in organometallic complexes has been reported previously for EXSY experiments (for example see ${ }^{[12]}$ ), their origin as being from metal relaxation has not been described or exploited, as far as we are aware. a)

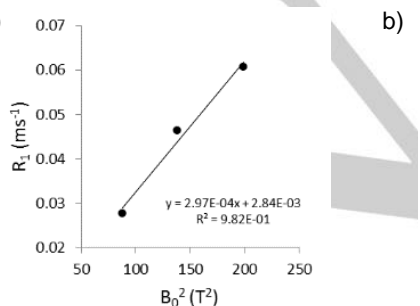

b)

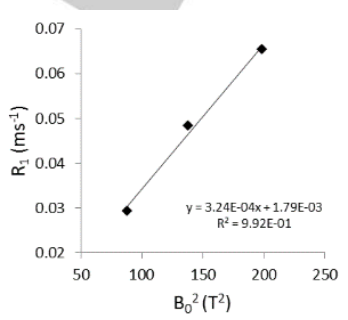

Figure 3. Magnetic field $\left(B_{0}\right)$ dependence of relaxation rates $\left(R_{1}=1 / T_{1}\right)$ of ${ }^{195} \mathrm{Pt}$ in complex $\mathbf{1}$ determined from (a) satellite exchange and (b) inversion recovery measurements.
In order to exploit this mechanism more fully, we sought to determine the longitudinal relaxation times for ${ }^{195} \mathrm{Pt}$ from these data to enable comparison with values determined from the classical inversion recovery experiment (which can be applied to ${ }^{195} \mathrm{Pt}$ as it is directly observable with conventional broad band NMR instrumentation).

A full consideration of the self-relaxation and crossrelaxation terms involving CSA and dipole-dipole (DD) mechanisms was employed to establish the relationship between the relaxation rates determined from direct observation (such as from saturation or inversion recovery experiments) and those determined from the satellite exchange experiments. Such an $a b$ initio analysis (described fully in Supporting Information) leads to the following expression for the longitudinal selfrelaxation rate of the $S$-spin $\left(R_{1}[S]\right)$ in a scalar-coupled $L-S$ spin pair, where we define $L$ to represent the high-y spin and $S$ the low-y (e.g. metal) spin:

$\mathrm{R}_{1}[S]=$

$$
-\frac{\Delta_{\mathrm{CSA}-\mathrm{S}}^{2}}{30} J\left(\tau_{\mathrm{C}}, \omega_{\mathrm{S}}\right)-\frac{\Delta_{\mathrm{DD}}^{2}}{360}\left[3 J\left(\tau_{\mathrm{C}}, \omega_{\mathrm{S}}\right)+J\left(\tau_{\mathrm{C}}, \omega_{L}-\omega_{\mathrm{S}}\right)+6 J\left(\tau_{\mathrm{C}}, \omega_{L}+\omega_{\mathrm{S}}\right)\right]
$$

where $\Delta_{\mathrm{CSA}}$ and $\Delta_{\mathrm{DD}}$ represent the magnitudes of the modulations in CSA and DD terms, respectively, and the $J\left(\tau_{c}, \omega\right)$ terms represent the associated spectral power densities (see Supporting Information). For the case of the satellite exchange cross-peaks, the longitudinal cross-relaxation rate between the satellites $\left(\mathrm{R}_{\mathrm{SE}}[L]\right)$ is:

$$
\begin{aligned}
& \mathrm{R}_{\mathrm{SE}}[L]= \\
& \frac{\Delta_{\mathrm{CSA}-\mathrm{S}}^{2}}{60} J\left(\tau_{\mathrm{C}}, \omega_{\mathrm{S}}\right)+\frac{\Delta_{\mathrm{DD}}^{2}}{720}\left[3 J\left(\tau_{\mathrm{C}}, \omega_{\mathrm{S}}\right)-J\left(\tau_{\mathrm{C}}, \omega_{\mathrm{L}}-\omega_{\mathrm{S}}\right)-6 J\left(\tau_{\mathrm{C}}, \omega_{\mathrm{L}}+\omega_{\mathrm{S}}\right)\right]
\end{aligned}
$$

In situations where the dipole-dipole cross-relaxation terms $\Delta_{\mathrm{DD}}$ may be considered negligible, these expressions reduce to:

$$
\mathrm{R}_{1}[S] \approx-\frac{\Delta_{\mathrm{CSA}-\mathrm{S}}^{2}}{30} J\left(\tau_{\mathrm{C}}, \omega_{\mathrm{S}}\right) \quad \text { and } \quad \mathrm{R}_{\mathrm{SE}}[L] \approx \frac{\Delta_{\mathrm{CSA}-\mathrm{S}}^{2}}{60} J\left(\tau_{\mathrm{C}}, \omega_{\mathrm{S}}\right)
$$

hence

$$
\mathrm{R}_{1}[S]=2 \mathrm{R}_{\mathrm{SE}}[L]
$$

Thus, provided CSA is the exclusive relaxation mechanism in operation for the low- $\gamma$ nucleus, it may be concluded that the longitudinal relaxation rate of the low- $\gamma$ spin $\left(\mathrm{R}_{1}[S]\right)$ is twice the relaxation rate determined from the satellite exchange measurements $\left(R_{\mathrm{SE}}[L]\right)$. Since in the case of complex 1 the CSA mechanism is dominant for ${ }^{195} \mathrm{Pt}$, the initial rates determined from the satellite exchange measurements of Figure 2 were adjusted to yield longitudinal $T_{1}$ values according to this relationship (Table 1). Hence, the initial rate at $9.4 \mathrm{~T}(400 \mathrm{MHz}$ $\left.{ }^{1} \mathrm{H}\right)$ was observed to be $14.6 \mathrm{~s}^{-1}$, which when doubled corresponded to a longitudinal relaxation rate of $29.2 \mathrm{~s}^{-1}$. This in turn yielded a $T_{1}$ value of $34.2 \mathrm{~ms}(1 /$ rate) at this field strength. Comparison with those $\mathrm{T}_{1}$ values determined directly from ${ }^{195} \mathrm{Pt}$ inversion recovery experiments (Supporting Information, Figures S3-5) at three different field strengths showed a very close correspondence (Table 1). Consistent with this, the results from the inversion recovery experiments showed an essentially identical linear dependence on $\mathrm{B}_{0}{ }^{2}$ (Fig 3b). These data demonstrate the robustness of the SE approach to the indirect determination of longitudinal spin relaxation rates when CSA is 
dominant. Parallel studies on ${ }^{31} \mathrm{P}$ indicated its relaxation also to be dominated by the CSA mechanism in complex 1 (Supporting Information Table S3 and Figure S6).

Table 1. ${ }^{195} \mathrm{Pt}$ Longitudinal relaxation time constants $\left(\mathrm{T}_{1}\right)$ as a function of magnetic field $\left(\mathrm{B}_{0}\right)$ measured indirectly via satellite exchange $(\mathrm{SE})$ in ${ }^{31} \mathrm{P}_{-}{ }^{31} \mathrm{P}$ EXSY and from direct measurement using inversion recovery (IR).

\begin{tabular}{l|lll}
\hline $\mathrm{B}_{0} / \mathrm{T}$ & ${\text { Initial rate } / \mathrm{s}^{-1}}$ & $\mathrm{~T}_{1} / \mathrm{ms}$ & $\mathrm{T}_{1} / \mathrm{ms}$ \\
$\left({ }^{1} \mathrm{H} / \mathrm{MHz}\right)$ & $(\mathrm{SE})$ & $(\mathrm{SE})$ & $(\mathrm{IR})$ \\
\hline $9.40(400)$ & 14.6 & 34.2 & 34.4 \\
$11.74(500)$ & 23.1 & 21.6 & 20.7 \\
$14.09(600)$ & 30.3 & 16.5 & 15.3 \\
\hline
\end{tabular}

To exemplify the application of this method to a more challenging system, we report our studies on the phosphorussilver(I) complexes $\mathbf{2}$ and $\mathbf{3}$ (Scheme 1) which are prepared and studied as a mixture (see Experimental Section). It was whilst studying the structural and dynamic properties of these complexes that we observed in their ${ }^{31} \mathrm{P}$ EXSY data cross-peaks that could not be rationalised based on classical consideration of chemical exchange processes or Overhauser effects and which ultimately led to the realisation that these cross-peaks reported indirectly on the relaxation properties of the scalar coupled silver nuclei, as explained below. Herein, we retain the term satellites to describe any metal-coupled peaks, regardless of isotope abundance.

These complexes are anticipated to have different geometrical properties, with complex 2 exhibiting a distorted tetrahedral geometry and complex 3 possessing a trigonal planar geometry. The ${ }^{1} \mathrm{~J}_{\mathrm{Ag}-\mathrm{P}}$ values for 2 of $226 \mathrm{~Hz}\left({ }^{107} \mathrm{Ag}\right)$ and $261 \mathrm{~Hz}\left({ }^{109} \mathrm{Ag}\right)$ are representative of $\mathrm{a} \mathrm{L}_{4} \mathrm{Ag}^{+}$tetrahedral centre whereas those for $3(408$ and $471 \mathrm{~Hz})$ are consistent with $\mathrm{L}_{2} \mathrm{Ag}^{+}$ coordination. ${ }^{[13]}$ It was therefore postulated that the relaxation properties of the silver centres might also reflect these geometrical differences. However, although both silver isotopes are spin $1 / 2$, they both have a very low magnetogyric ratio and poor sensitivity which rendered their direct observation impossible with available sample quantities. Most standard NMR probes are also unable to operate at sufficiently low frequencies for these isotopes, making the satellite exchange approach attractive for studying this system.

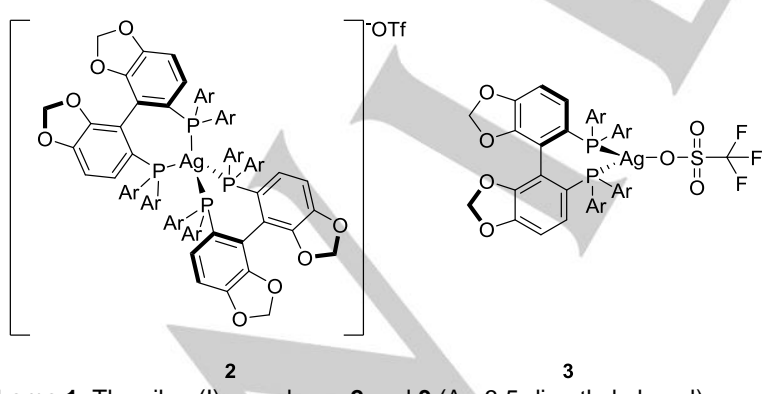

Scheme 1. The silver(I) complexes 2 and 3 ( $A r=3,5$-dimethyl phenyl).

Initial ${ }^{31} \mathrm{P}_{-}{ }^{31} \mathrm{P}$ EXSY experiments were performed at $233 \mathrm{~K}$ (the temperature at which complex 2 was being employed for catalytic transformations) in order to determine whether $\mathbf{2}$ and $\mathbf{3}$ existed in dynamic equilibrium. These experiments demonstrated this was not the case as exchange peaks were not observed between them (Fig 4a). However, chemical exchange was observed for the labile trigonal planar complex 3 arising from dissociation-association of the bisphosphine ligand with the metal. This behaviour was characterised by quadrant cross-peaks for the trigonal species, and specifically by those corresponding to a change in metal isotope for the phosphine adduct (cross-peaks labelled $A-B, B-A, A^{*}-B^{*}, B^{*}-A^{*}, B-A^{*}$ and $A-B^{*}$ in Figure $4 b$ ). In contrast, these metal exchange crosspeaks were not observed for the non-labile complex 2 at $233 \mathrm{~K}$. On further cooling of the sample to $203 \mathrm{~K}$ these metal exchange cross-peaks also disappeared for trigonal complex $\mathbf{3}$, suggesting this process had become too slow to be detected under these conditions (Fig. 5). Significantly, for both complexes 2 and $\mathbf{3}$, cross-peaks correlating satellites arising from a single silver isotope (labelled $A-A^{*}$ and $B-B^{*}$ in Figs $4 b$ and 5 ) remain under these conditions. Although in principle it would be possible for these to be generated by ligand dissociation, ${ }^{\ddagger}$ this process does not apply for non-labile complex 2 or for complex $\mathbf{3}$ when at 203 $\mathrm{K}$ (where all other possible ligand exchange peaks are not seen). Hence, under these conditions, the $A-A^{*}$ and $B-B^{*}$ type crosspeaks originate from satellite exchange caused by longitudinal relaxation of the silver nucleus in both complexes, analogous to that described for the platinum system above.

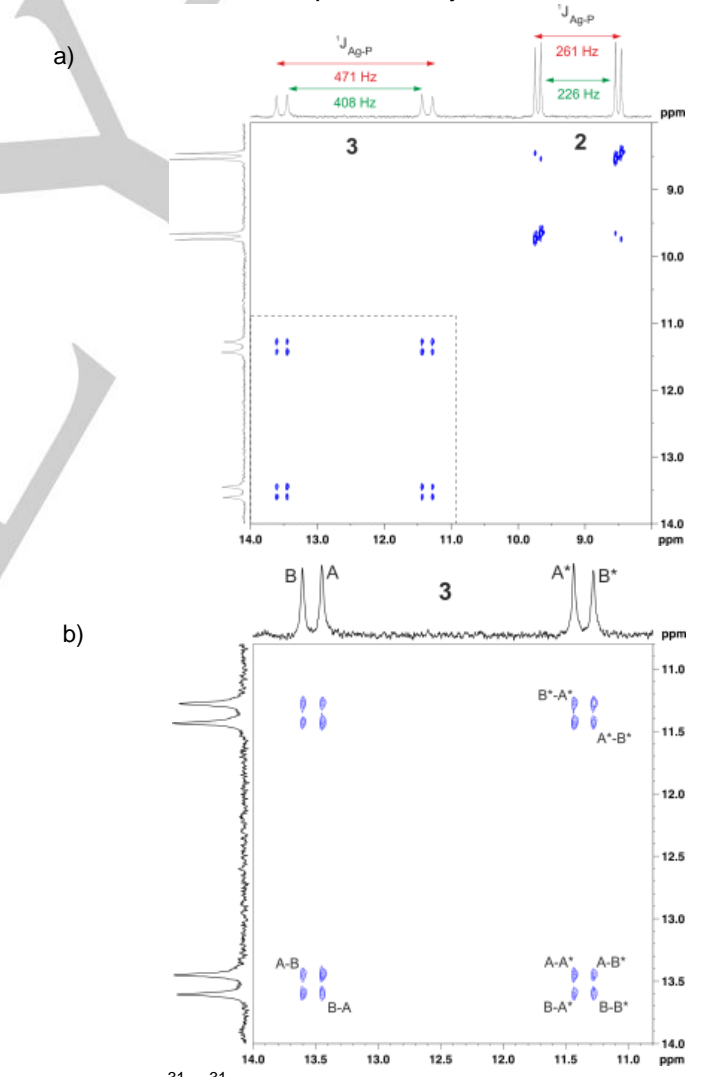

Figure 4. a) ${ }^{31} \mathrm{P}_{-}{ }^{31} \mathrm{P}$ EXSY of complexes 2 and 3 recorded at $233 \mathrm{~K}\left(\mathrm{t}_{\mathrm{mix}}=800\right.$ $\mathrm{ms}, \mathrm{CDCl}_{3}$ ); one-bond $\mathrm{Ag}-\mathrm{P}$ coupling constants for each metal isotope (red ${ }^{109} \mathrm{Ag}$, green ${ }^{107} \mathrm{Ag}$ ) are indicated on the $1 \mathrm{D}$ phosphorus spectrum shown above. b) Region of EXSY spectrum shown boxed in (a) highlighting the trigonal planar complex 3 . Labelled cross-peak are discussed in the main text.

\footnotetext{
$\$$ The $A-A^{*}$ and $B-B^{*}$ peaks could arise if the bisphosphine ligand dissociated and then re-associated with another metal ion of the same silver isotope but with the opposite spin state.
} 
Following the protocol outlined above, the normalised cross-peak build-up profiles were measured at $203 \mathrm{~K}$ for the silver complexes (Fig 6). The initial slopes yielded corresponding $\mathrm{T}_{1}$ longitudinal relaxation time constants for ${ }^{109} \mathrm{Ag} /{ }^{107} \mathrm{Ag}$ of 4.67/4.59 s and 0.49/0.56 s for complexes 2 and 3 respectively from the satellite exchange analysis, assuming CSA to be the dominant relaxation mechanism. The rather limited literature information on silver relaxation ${ }^{[6]}$ suggests CSA is indeed likely to be dominant in this regard, and our DFT derived estimates also support this (see Supporting Information). The ten-fold difference in relaxation rates correlates qualitatively with expectations from the differing geometries of the complexes. Thus, the higher symmetry in complex 2 correlates with a greater relaxation time due to the smaller CSA in the distorted tetrahedral environment of the metal relative to the lower symmetry and hence greater CSA experienced in complex 3 . To assess this quantitatively we computed chemical shielding tensors for DFT optimised structures of complexes $\mathbf{2}$ and $\mathbf{3}$, and from these we estimated correlation times and longitudinal relaxation rates for their silver nuclei at $203 \mathrm{~K}$ (see Supporting Information). These calculations predict a four-fold difference in the magnitudes of spin relaxation rates which, given the estimates involved, $\S$ is comparable to that observed experimentally. The measured $\mathrm{T}_{1}$ values are considerably shorter than those reported for isolated ions at low field, ${ }^{[8]}$ and likely reflect the influence of higher $\mathrm{B}_{0}$ values and the lowtemperatures, hence increased correlation times, used in our studies. Indeed, for low- $\gamma{ }^{57} \mathrm{Fe} \mathrm{T}_{1}$ values of $1 \mathrm{~s}$ have been reported for ferrocene at $9.4 \mathrm{~T}^{[3 \mathrm{~b}]}$ and as low as $17 \mathrm{~ms}$ in the protein carbonmonoxymyoglobin at $8.45 \mathrm{~T}^{[14]}$

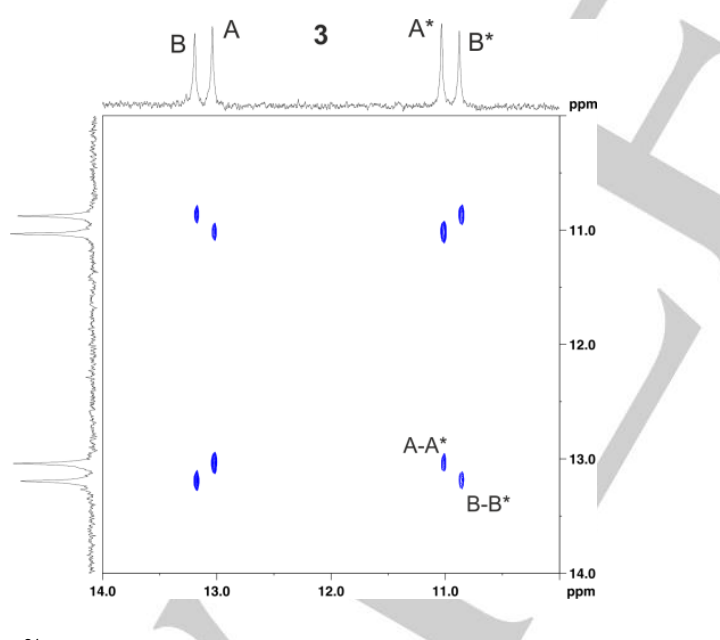

Figure $5 .{ }^{31} \mathrm{P}_{-}{ }^{31} \mathrm{P}$ EXSY for the mixture of complexes 2 and 3 recorded at 203 $\mathrm{K}\left(\mathrm{t}_{\text {mix }}=800 \mathrm{msec} ; \mathrm{CD}_{2} \mathrm{Cl}_{2}\right.$ ) showing an expanded region for complex 3 in which only satellite exchange cross-peaks $A-A^{*}\left({ }^{107} \mathrm{Ag}\right)$ and $B-B^{*}\left({ }^{109} \mathrm{Ag}\right)$ are observed; no exchange arising from ligand dissociation is seen.

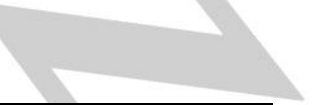

$\S$ DFT calculations provide only rough estimates of $T_{1}$. DFT shielding anisotropies for silver have uncertainties likely as high as $30 \%$, and the hydrodynamic estimates of the rotational correlation times did not account for possible solvent coordination. As the relaxation rate depends on the product of shielding anisotropy squared and the rotational correlation time, the uncertainties amplify, and the results should only be viewed as order of magnitude estimates.

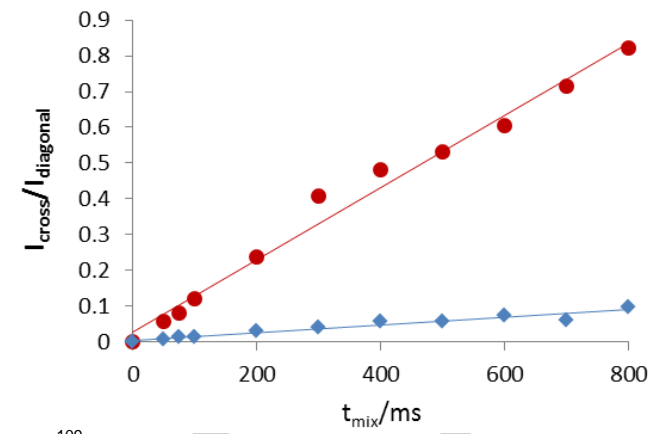

Figure 6. ${ }^{109} \mathrm{Ag}$ Satellite exchange cross-peak profiles $\left(\mathrm{I}_{\text {cross }} / \mathrm{I}_{\text {diagonal }}\right)$ from ${ }^{31} \mathrm{P}$ ${ }^{31} \mathrm{P}$ EXSY experiments at $203 \mathrm{~K}\left(\mathrm{CD}_{2} \mathrm{Cl}_{2}\right)$ for tetrahedral complex 2 (blue triangles) and trigonal complex 3 (red circles). See Figs S15-16 for ${ }^{107} \mathrm{Ag}$ data.

The use of EXSY NMR for the elucidation of kinetic parameters relating to chemical exchange processes is well documented. ${ }^{[15]}$ However, the indirect extraction of relaxation data of a low- $y$, insensitive nuclide from the EXSY spectra of a scalar-coupled partner has hitherto been unreported, as far as we are aware. Herein, we utilise the phenomenon of satellite exchange occurring during the EXSY experiment to report on the relaxation behaviour of metal nuclei, here exemplified using ${ }^{195} \mathrm{Pt}$ as a model system and ${ }^{107 / 109} \mathrm{Ag}$ as a more challenging application. Diamagnetic spin-1/2 transition metals typically have CSA dominated relaxation (unless in highly symmetric environments) and the indirect measurement of their longitudinal relaxation time constants $\left(T_{1} \mathrm{~s}\right)$ becomes possible without the need to directly observe, or even pulse on, the low- $y$ nuclide. This is possible provided its longitudinal relaxation rate is comparable to or faster than that of the directly observed nucleus. This approach employing satellite exchange NMR spectroscopy (SENSY) offers the potential to extend relaxation studies of organometallic systems to include many transition metals that might otherwise prove impractical or even impossible to measure.

\section{Acknowledgements}

We thank EPSRC for funding via a studentship to GPR and grant EP/N006895/1 to IK.

Keywords: NMR Spectroscopy • Relaxation • SENSY• Silver • Transition metals

T. D. W. Claridge, High-Resolution NMR Techniques in Organic Chemistry, 3rd ed., Elsevier, Boston, 2016.

[2] T. C. Farrar, I. C. Locker, J. Chem. Phys. 1987, 87, 3281-3287.

[3] a) B. M. Still, P. G. A. Kumar, J. R. Aldrich-Wright, W. S. Price, Chem. Soc. Rev. 2007, 36, 665-686; b) W. von Philipsborn, Pure Appl. Chem. 1986, 58, 513-528; c) R. Benn, H. Michael Büch, R.-D. Reinhardt, Magn. Reson. Chem. 1985, 23, 559-564. 
[4] a) W. von Philipsborn, Chem. Soc. Rev. 1999, 28, 95105; b) R. Benn, A. Rufińska, Angew. Chem. Int. Ed. 1986, 25, 861-881.

[5] J. M. Ernsting, S. Gaemers, C. J. Elsevier, Magn. Reson. Chem. 2004, 42, 721-736.

[6] G. H. Penner, X. Liu, Prog. Nucl. Magn. Reson. Spectrosc. 2006, 49, 151-167.

[7] R. Villanneau, S. Renaudineau, P. Herson, K. Boubekeur, R. Thouvenot, A. Proust, Eur. J. Inorg. Chem. 2009, 2009, 479-488.

[8] H. Pfister, A. Schwenk, D. Zeller, J. Magn. Reson. 1986, 68, 138-145.

[9] C. E. Keefer, R. D. Bereman, S. T. Purrington, B. W. Knight, P. D. Boyle, Inorg. Chem. 1999, 38, 2294-2302.

[10] a) J.-Y. Lallemand, J. Soulie, J.-C. Chottard, J. Chem. Soc., Chem. Commun. 1980, 436-438; b) P. S. Pregosin, Coord. Chem. Rev. 1982, 44, 247-291; c) R. Benn, R.-D. Reinhardt, A. Rufińska, J. Organomet. Chem. 1985, 282, 291-295.

[11] I. M. Ismail, S. J. S. Kerrison, P. J. Sadler, Polyhedron 1982, $1,57-59$.

[12] a) A. Habtemariam, J. A. Parkinson, N. Margiotta, T. W. Hambley, S. Parsons, P. J. Sadler, J. Chem. Soc., Dalton Trans. 2001, 362-372; b) H. Bircher, B. R. Bender, W. von Philipsborn, Magn. Reson. Chem. 1993, 31, 293-298.

[13] a) E. L. Muetterties, C. W. Alegranti, J. Am. Chem. Soc. 1970, 92, 4114-4115; b) N. Momiyama, H. Yamamoto, $J$. Am. Chem. Soc. 2004, 126, 5360-5361.

[14] H. C. Lee, J. K. Gard, T. L. Brown, E. Oldfield, J. Am. Chem. Soc. 1985, 107, 4087-4088.

[15] C. L. Perrin, T. J. Dwyer, Chem. Rev. 1990, 90, 935-967. 


\section{COMMUNICATION}

An approach to the indirect measurement of nuclear spin relaxation rates of low-magnetogyric ratio $(\mathrm{r})$ nuclei is described. The method does not require the observation of, or even the ability to provide radio-frequency pulses to, the low-y nucleus, but requires this to be scalar coupled to an NMR observable nucleus, such as ${ }^{31} \mathrm{P}$ or ${ }^{1} \mathrm{H}$.

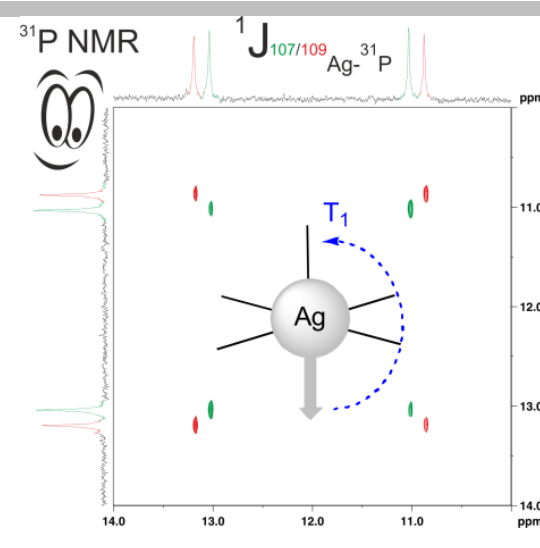

Gerard P. Robertson, Barbara Odell, Ilya Kuprov, Darren J. Dixon, and Tim D. W. Claridge*

Page No. - Page No.

Title 